\title{
Interactions of combined bile acids on hepatocyte viability: cytoprotection or synergism
}

\author{
Anabela P. Rolo a , Carlos M. Palmeira ${ }^{\text {a }}$, Kendall B. Wallace ${ }^{\text {b,* }}$

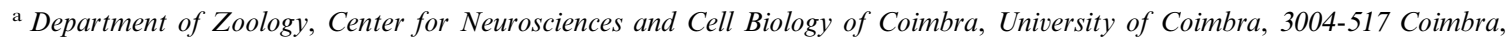 \\ Portugal \\ ${ }^{\mathrm{b}}$ Department of Biochemistry and Molecular Biology, University of Minnesota School of Medicine, 10 University Drive, Duluth, \\ MN 55812, USA
}

Received 27 July 2001; received in revised form 22 October 2001; accepted 23 October 2001

\begin{abstract}
Cholestasis results from hepatocyte dysfunction due to the accumulation of bile acids in the cell, many of which are known to be cytotoxic. Recent evidence implicates competitive antagonism of key cytotoxic responses as the mechanism by which certain therapeutic bile acids might afford cytoprotection against cholestasis. In this work, we compare the relative cytotoxicity of bile acids in terms of dose- and time-dependence. To better elucidate the controversy related to the therapeutic use of ursodeoxycholate (UDCA) in cholestatic patients, we also evaluated the effects of bile acid combinations. Viability of Wistar rat hepatocytes in primary culture was measured by LDH leakage after 12 and $24 \mathrm{~h}$ exposure of cells to the various bile acids. All unconjugated bile acids caused a dose-dependent decrease in cell viability. The tauro- and glyco-conjugates of chenodeoxycholate (CDCA) and UDCA were all less toxic than the corresponding unconjugated form. Although relatively non-toxic, UDCA caused synergistic cell killing by lithocholate (LCA), CDCA, glyco-CDCA (GCDC) and tauro-CDCA (TCDC). Glycoursodeoxycholate decreased the toxicity of GCDC, but potentiated the toxicity of unconjugated CDCA and LCA. The tauro-conjugate of UDCA had no significant effect. These data suggest that at cholestatic concentrations, bile acid-induced cell death correlates with the degree of lipophilicity of individual bile acids. However, these results indicate that the reported improvement of biochemical parameters in cholestatic patients treated with UDCA is not due to a direct effect of UDCA on hepatocyte viability. Therefore, any therapeutic effect of UDCA must be secondary to some other process, such as altered membrane transport or nonparenchymal cell function. (C) 2002 Elsevier Science Ireland Ltd. All rights reserved.
\end{abstract}

Keywords: Hepatocytes; Bile acids; Ursodeoxycholate; Mixtures; Cholestasis

\section{Introduction}

Bile acids are sterol-derived, potentially cyto-

* Corresponding author. Tel.: + 1-218-726-8899; fax: + 1218-726-8014.

E-mail address: kwallace@d.umn.edu (K.B. Wallace). toxic compounds synthesized and secreted by hepatic epithelial cells into the bile canaliculus (Plaa et al., 1982). During cholestasis (an impairment in 
bile flow) bile acids accumulate in the hepatocyte (Greim et al., 1972). This accumulation of cytotoxic bile acids is thought to cause hepatocyte necrosis contributing to the pathogenesis of the cholestatic disease process and the development of liver cirrhosis and liver failure (Kaplan, 1994). Mechanisms implicated in the toxicity of bile acids include stimulation of lipid peroxidation (Sokol et al., 1993) and induction of mitochondrial dysfunction (Schaffner et al., 1971; Krahenbuhl et al., 1992; Gores et al., 1998; Rolo et al., 2000).

Due to their membrane-active, detergent-like properties, the cytotoxicity of bile acids has been associated with the degree of lipophilicity of the different molecular species (Scholmerich et al., 1984; Delzenne et al., 1992). Ursodeoxycholate (UDCA) is more hydrophilic than the primary, dihydroxy bile acid chenodeoxycholate (CDCA). Lithocholate (LCA), a secondary monohydroxylated bile acid, is the most lipophilic of all bile acids (Sarbu et al., 2001). In humans, bile acids are conjugated to glycine and taurine, with the glycine conjugates predominating (Hofmann, 1984). Conjugation decreases the lipophilicity and renders the bile acids less cytotoxic (Sarbu et al., 2001).

Not all bile acids are cytotoxic and minor changes in bile acid structure can markedly alter the biological activity (Scholmerich et al., 1984). CDCA is the toxic bile acid most widely implicated in cholestatic liver injury (Greim et al., 1972). In contrast, UDCA (the $\beta$-epimer of CDCA) has been shown to reduce serum liver enzymes in chronic liver diseases, although not in all patients (Poupon et al., 1994). Ursodeoxycholic acid (UDCA) is considered to be an effective treatment for primary biliary cirrhosis and other cholestatic liver diseases in humans (Beuers et al., 1998), such as intrahepatic cholestasis of pregnancy. Serum liver tests and histopathological suggest improvement of liver function in patients treated with UDCA. In most of the cases, UDCA shows a favorable effect on biochemical indices (van Hoogstraten et al., 1999) but not on symptoms or the progression of histological stage (Neuberger, 2000). Indeed, the recent report by Neuberger 'URSO - panacea or placebo?' draws attention to the necessity to re-examine the therapeutic benefit of UDCA.
The nature of this apparent cytoprotection is still unclear. Although some studies reported bile acids effects on cell viability (Delzenne et al., 1992; Spivey et al., 1993; Hillaire et al., 1995), no systematic study has been carried out. With growing debate regarding the effectiveness of UDCA and conjugates in patients with cholestasis, several important questions are raised, one of which is whether the observed effects of UDCA in vivo are related to a direct effect at the hepatocyte level. In order to address this question, we evaluated the effects of UDCA and conjugates on hepatocyte cell viability, either alone or in combination with other bile acids.

\section{Materials and methods}

\subsection{Materials}

UDCA, LCA, glycoursodeoxycholate (GUDC), CDCA and conjugates, were purchased from Sigma Chemical Co. (St. Louis, MO). Tauroursodeoxycholate (TUDC), as sodium salt, was obtained from Cal Biochem (La Jolla, CA). UDCA and LCA were dissolved in ethanol, all others in water. Lactate dehydrogenase (LDH) was purchased from Sigma and Collagenase type 2 was from Worthington (Freehold, NJ). All other chemicals were of analytical grade and obtained from the standard commercial sources. A Labsystems type 374 plate-reader was used for all measurements of fluorescence intensity.

\subsection{Animals}

Male Sprague-Dawley rats (Harlan Laboratories, Madison, WI) weighing $198 \pm 6$ g were maintained in AAALAC-accredited, climate-controlled facilities and allowed free access to food (Purina Chow) and water.

\subsection{Rat hepatocyte isolation and maintenance in cell culture}

Hepatocytes were isolated according to a modified procedure of Seglen (1976). The main alterations were that perfusion of the liver with 
collagenase was performed in situ and after digestion, cells were dispersed in RPMI growth medium. Cells were plated on 12-well plates at a density of $0.6 \times 10^{6}$ cells per well. Culture medium was Rosewell Park Memorial Institute (RPMI) medium supplemented with $5 \%$ fetal bovine serum, $1 \mu \mathrm{M}$ insulin, $100 \mu \mathrm{M}$ hydrocortisone sodium succinate, gentamicin $(50 \mathrm{mg} / \mathrm{ml})$ and 15 $\mathrm{mM}$ HEPES ( $\mathrm{pH} 7.4$ ). Cells were maintained in an incubator at $37{ }^{\circ} \mathrm{C}, 5 \% \mathrm{CO}_{2}$ and humidified atmosphere. After allowing 2-3 h for the cells to attach, medium was replaced and the cells left for 18-24 h. The culture medium was then replaced and bile acids were added. Treatment was carried out under the same incubation conditions for up to $24 \mathrm{~h}$.

\subsection{Cell viability assay}

Cell viability was determined fluorometrically by estimating release of LDH into media (Moran and Schnellmann, 1996). The determination of LDH activity is based on the reduction of pyruvate to lactate, as monitored by the decrease in $\mathrm{NADH}$ fluorescence at $450 \mathrm{~nm}$ wavelength with excitation wavelength $355 \mathrm{~nm}$. Fresh reaction solution was made by mixing $0.4 \mathrm{ml}$ of $16.2 \mathrm{mM}$ pyruvate with $10 \mathrm{ml}$ of $0.2 \mathrm{mM}$ NADH in phosphate buffer ( $\mathrm{pH}$ 7.5). After incubation of hepatocytes with bile acids for 12 or $24 \mathrm{~h}, 5 \mu \mathrm{l}$ of the cell-free supernatant was added to $200 \mu$ of fresh assay solution to initiate the reaction. Total cellular LDH was determined by lysing the cells after freezing in a solution of $0.1 \mathrm{M}$ sodium phosphate buffer ( $\mathrm{pH}$ 7.0) The amount of LDH released into the media was expressed as a percentage of total LDH. Using this method, control cell viability was $72.1 \pm 9.2 \%$ after $24 \mathrm{~h}$ of incubation.

\subsection{Statistical analysis}

All data are expressed as the mean \pm S.E.M. for three to four independent experiments. Statistical evaluation was performed using the two-tailed paired Student's $t$-test. A $P$ value $<0.05$ was considered statistically significant.

\section{Results and discussion}

Bile acids are amphipathic, endogenous steroid compounds that possess detergent properties, which at high concentrations have deleterious effects upon cell membranes (Scholmerich et al., 1984). However, at lower concentrations $(\approx 50$ $\mu \mathrm{M})$, bile acids may alter cell function by interfering with intracellular signaling mechanisms (Rust et al., 2000) and mitochondrial membrane permeability (Gores et al., 1998; Rolo et al., 2000). Regardless, cholestasis due to accumulation of bile acids has been thought to reflect the direct cytotoxicity of the individual bile acids (Kaplan, 1994).

In the present work, cell viability was investigated to assess the relative potency of bile acids on rat hepatocytes in primary culture. The structures of the individual bile acids included in this investigation are illustrated in Table 1. Cytotoxicity of individual bile acids was both time and concentration dependent and corresponded to their lipophilic character. Upon $12 \mathrm{~h}$ of exposure, a decrease in cell viability was observed most dramatically for CDCA and LCA. Hepatocyte viability at $12 \mathrm{~h}$ was $70 \pm 9 \%$ for $150 \mu \mathrm{M}$ CDCA,

Table 1

Relationship between bile acid structure and their polarity/ acidity versus lipophilicity (Sarbu et al., 2001)

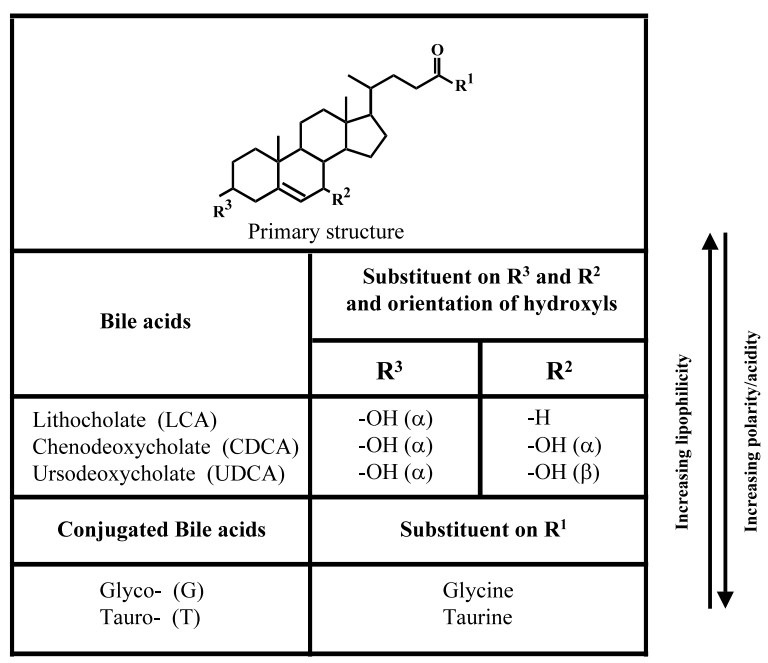




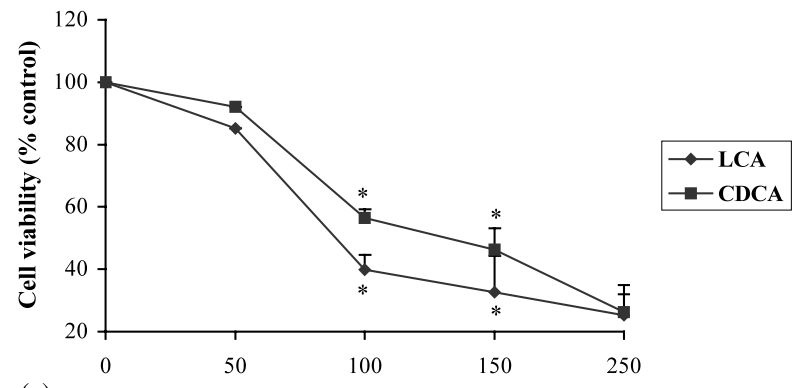

(a)

Bile acid concentration $(\mu \mathrm{M})$
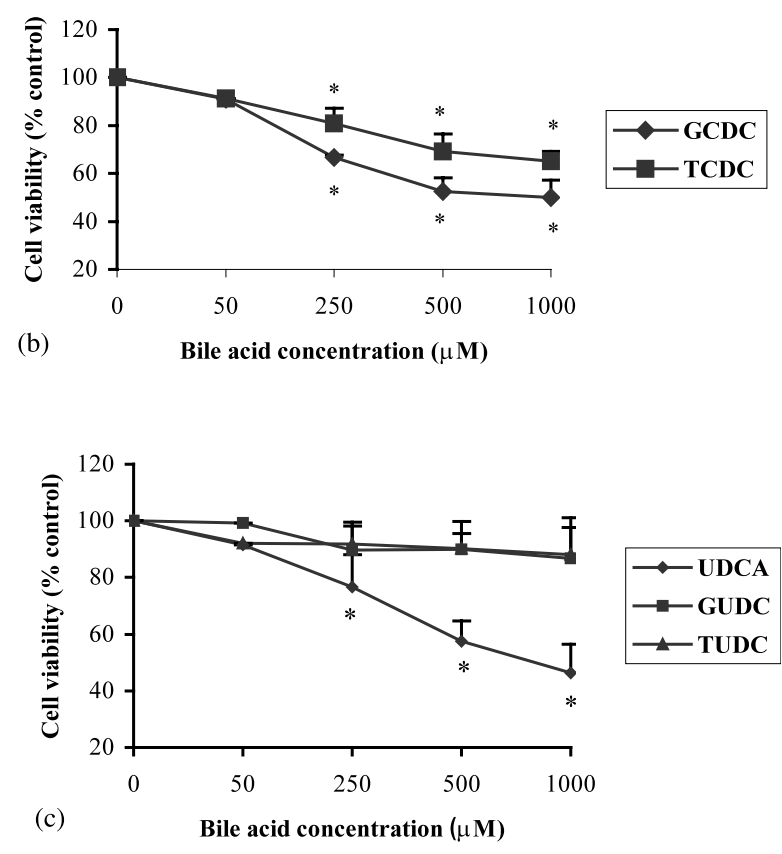

Fig. 1. Changes in cell viability following $24 \mathrm{~h}$ incubation with different concentrations of bile acids. Cellular viability was determined fluorometrically by estimating the release of LDH into media. Total cellular LDH was determined after lysing cells; the amount of $\mathrm{LDH}$ released into media at any given time during the incubation was expressed as the percentage of total LDH. Percent viability was obtained by comparing with control cell viability, considered as $100 \%$. Data represent the mean \pm S.E.M. of measurements from three separate animals. *Values statistically different from control $(P<0.05)$.

$59 \pm 9 \%$ for $150 \mu \mathrm{M}$ LCA and $77 \pm 10 \%$ for 500 $\mu \mathrm{M}$ taurochenodeoxycholate (TCDC). Exposure to the lipophilic bile acids CDCA and LCA for 24 $\mathrm{h}$ resulted in a significant enzyme release even at low concentrations (Fig. 1A), whereas hydrophilic bile acids, UDCA, TCDC and glycochenodeoxy- cholate (GCDC) required higher concentrations to cause a significant decrease in cell viability in $24 \mathrm{~h}$ (Fig. 1B, C). The fact that conjugation with either glycine or taurine decreased cytotoxicity of CDCA agrees with the observations made by others (Scholmerich et al., 1984). In contrast, Spivey et al. (1993) reported that at a concentration of $250 \mu \mathrm{M}$, GCDC was more toxic than either CDCA or TCDC. Significant differences in the experimental procedure may explain the discrepancy. For instance, Spivey et al. (1993) worked with hepatocytes after only $2 \mathrm{~h}$ in culture.

Cell viability after $24 \mathrm{~h}$ exposure to the glycoand tauro-conjugated forms of UDCA was also tested. Both glyco- (GUDC) and tauroursodeoxycholate (TUDC) were found to have no significant effect on LDH release (Fig. 1C), whereas unconjugated UDCA at $500 \mu \mathrm{M}$ decreased cell viability to $58 \pm 10 \%$ control.

The next set of experiments was designed to assess the putative hepatoprotective effects of UDCA and derivatives against the cytotoxicity of more lipophilic bile acids implicated in cholestasis. Based on the results of the first set of experiments, $250 \mu \mathrm{M}$ UDCA was selected as the concentration for use in these studies $(\approx 25 \%$ cell killing after $24 \mathrm{~h}$ incubation). The non-toxic tauro- and glycoursodeoxycholate were used at $500 \mu \mathrm{M}$. Fig. 2(A,B) shows that co-incubation of rat hepatocytes for $24 \mathrm{~h}$ in primary culture with both UDCA and either $100 \mu \mathrm{M}$ LCA, $150 \mu \mathrm{M}$ CDCA, $500 \mu \mathrm{M}$ GCDC or $500 \mu \mathrm{M}$ TCDC, resulted in a significant increase in enzyme leakage compared to the bile acids alone. These data demonstrate that contrary to in vivo exposure, there is no cytoprotection by UDCA against bile acid-induced hepatocyte lethality in vitro. In fact, UDCA synergized the cytotoxicity of all other bile acids in hepatocyte cell culture. Similar results were observed for $12 \mathrm{~h}$ co-incubations (data not shown). Our results agree with those of Hillaire et al. (1995) who reported concentration-dependent increases in LDH leakage by CDCA $(100-500$ $\mu \mathrm{M})$ in human hepatocytes in primary culture. Additionally, Hillaire et al. observed that TUDC and UDCA (at the same concentrations used in our study) did not have a protective effect when added concomitantly with CDCA (Hillaire et al., 
1995). A report by Pazzi et al. (1997), however, indicates that addition of UDCA significantly reduced the hepatotoxic effect of the lipophilic bile acid, deoxycholate but not chenodeoxycholate. These studies were made with freshly isolated rat hepatocytes suspended on a resin column and perfused with different concentrations of bile acids (Pazzi et al., 1997). Galle et al. (1990) also
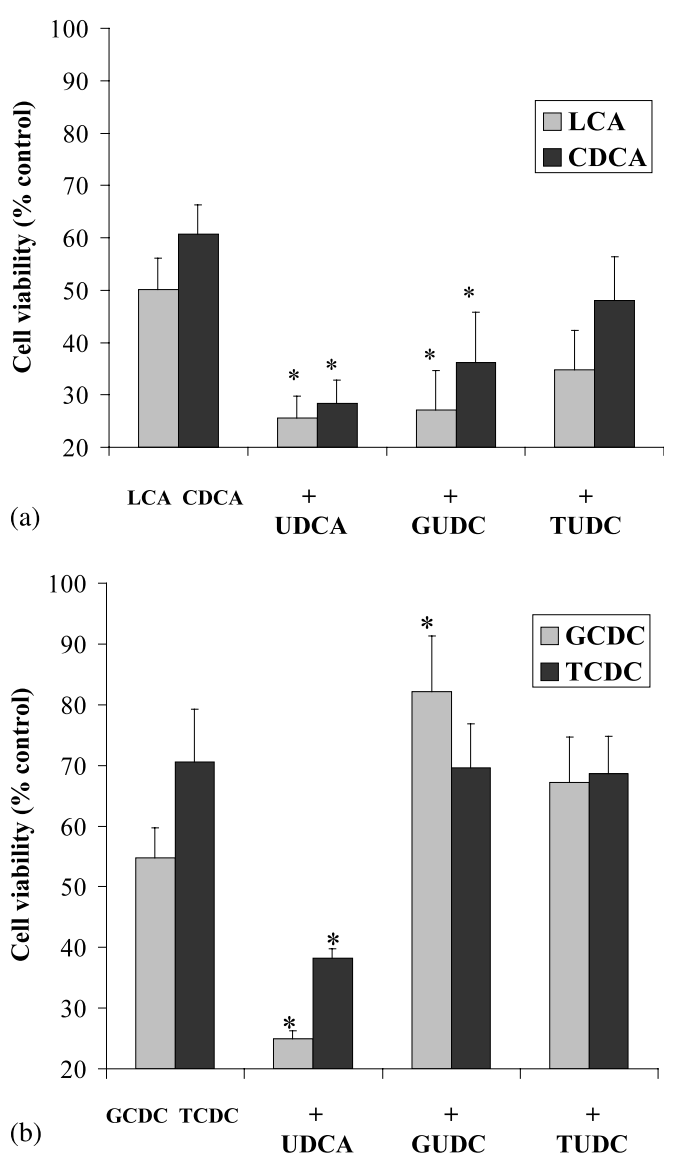

Fig. 2. Cell viability after exposure to combinations of bile acids: UDCA, GUDC or TUDC with LCA, CDCA, GCDC or TCDC. Cellular viability was determined fluorometrically by estimating the release of $\mathrm{LDH}$ into media. Total cellular LDH was determined after lysing the cells; the amount of LDH released into media at any given time during the incubation was expressed as the percentage of total LDH. Percent viability was obtained by comparing with control cell viability, considered as $100 \%$. Data represent the mean \pm S.E.M. of measurements from four separate animals. *Values statistically different when compared to cells exposed just to CDCA, LCA, GCDC or TCDC $(P<0.05)$. reported a decrease of toxicity of GCDC by UDCA in primary human hepatocytes.

We also evaluated the potential for TUDC to be cytoprotective in combination with cytotoxic bile acids. In this case, TUDC was found to have no significant effect on hepatocyte killing by LCA, CDCA, GCDC or TCDC (Fig. 2A, B). In a previous report, Heuman et al. (1991) described that the hepatotoxicity of lipophilic bile acids was reduced in the presence of TUDC. However, this study was aimed at evaluating the immediate effects of therapeutic bile acids at the cellular level. Primary monolayer cultures of adult rat hepatocytes were incubated for 1-240 min with varying concentrations of the different bile acids (Heuman et al., 1991). This contrasts with the much more prolonged exposure of the present investigation.

GUDC elicited mixed effects on cell viability when added concomitantly with the other bile acids. Whereas GUDC did not alter the cytotoxicity of TCDC (Fig. 2B), it was cytoprotective against GCDC (Fig. 2B), but potentiated the cytotoxicity of both LCA and CDCA (Fig. 2A).

The differential effect of tauro- and glyco-conjugated bile acids may reflect slight differences in physical-chemical properties. For example, amidation is known to significantly reduce the cytotoxicity of the more lipophilic forms of unconjugated bile acids, both in hepatocytes (Scholmerich et al., 1984) and bile duct cells. As such, the tauro-conjugated derivatives are less effective than the glyco-conjugated species. The basis for this distinction is not known and it may be related to intracellular transport. Alternatively, taurine- and glycine-conjugated forms may differentially activate signaling cascades leading to the activation or repression of specific mechanisms controlling cell death and survival (Spivey et al., 1993; Que et al., 1999; Rust et al., 2000).

In the present work, we observed a strong correspondence between bile acid toxicity and their degree of lipophilicity, the most important feature concerning biological activity. A recent report (Sarbu et al., 2001) concerning the lipophilic character of bile acids and their tauroand glyco-conjugates showed that the main difference between the three subgroups of compounds is their polarity and acidity (Table 1). Glyco-con- 
jugates share many of the same properties as the free bile acids. However, the tauro-conjugates are more polar and acidic and thereby substantially less lipophilic. This could explain the similar activities of the unconjugated and glycine conjugated bile acids against hepatocyte cell killing, but with dissimilar behavior of the taurine conjugates. Although we can only speculate on a probable explanation for the observed synergism, the greater than additive effect of UDCA and the potentiation of cell death by GUDC (when combined with LCA, CDCA, GCDC and TCDC) may reflect non-linear dose-response, whether it be by a detergent/membrane damaging effect or at level of cell signal/cell function.

UDCA is the only approved treatment for chronic cholestatic liver disease, but its effects on disease progression are not completely clarified. A marked improvement in biochemical parameters is frequently reported (van Hoogstraten et al., 1999); however, this improvement is not reflected in the overall rate of progression of histological stage associated with chronic cholestatic liver disease or the clinical symptoms of the patients (Neuberger, 2000). In order to provide new insights into the understanding of the effects of therapeutic bile acids, in vitro studies have been done. Several reports described a role for the putative beneficial effect of UDCA exerted at the level of mitochondrial function, where UDCA prevents the impairment of mitochondrial function induced by toxic bile acids (Gores et al., 1998; Rodrigues et al., 1998). It has also been reported that UDCA could exert a cytoprotective action related to oxidative injury and antioxidant systems (Mitsuyoshi et al., 1999). However, other studies do not show cytoprotection by UDCA against toxic bile acids at the level of mitochondria or cell function (Krahenbuhl et al., 1994; Hillaire et al., 1995; Rolo et al., 2000). The report by Krahenbuhl et al. (1994) described that UDCA, but not TUDC, decreased the toxicity of lipophilic bile acids at the level of mitochondrial electron transport chain, up to a concentration of $100 \mu \mathrm{M}$. However, at higher concentrations, UDCA increased bile acid-induced mitochondrial toxicity (Krahenbuhl et al., 1994). Additionally, our previous data (Rolo et al., 2000) also demon- strated no role for UDCA in preventing mitochondrial dysfunction induced by hydrophobic bile acids. In contrast, UDCA increased impairment of mitochondrial function, which agrees with the observed effects on hepatocytes in primary culture.

In fact, rather than cytoprotection, we observed that UDCA caused synergistic cell killing by all other bile acids. This then provides compelling evidence that the reported improvement of biochemical parameters in patients with cholestasis does not reflect an interaction of the bile acids directly on hepatocyte viability and must therefore reflect some other level of interaction, such as membrane transport or non-parenchymal cell function. Generation of bile flow is a regulated process that depends on the coordinated action of a number of transporter proteins in the sinusoidal and canalicular domains of the hepatocyte. The expression and function of these transport proteins are known to be significantly altered during cholestasis (Muller and Jansen, 1997). Since UDCA enriches the bile in cholestatic patients receiving treatment (Lindor et al., 1998), a preferential uptake of UDCA by the transporters could explain the observed improvement in biochemical indices (van Hoogstraten et al., 1999).

\section{Acknowledgements}

A.P. Rolo is a recipient of a PRAXIS Grant XXI/21454/99 from the Fundacao para a Ciencia e Tecnologia (FCT), Lisbon, Portugal. C.M. Palmeira was supported by the Luso-American Foundation (Lisbon, Portugal).

\section{References}

Beuers, U., Boyer, J.L., Paumgartner, G., 1998. Ursodeoxycholic acid in cholestasis: Potential mechanisms of action and therapeutic applications. Hepatology 28, 1449-1453.

Delzenne, N.M., Calderon, P.B., Taper, H.S., Robertroid, M.B., 1992. Comparative hepatotoxicity of cholic acid, deoxycholic acid and lithocholic acid in the rat: in vivo and in vitro studies. Toxicol. Lett. 61, 291-304.

Galle, P.R., Theilmann, L., Raedsch, R., Otto, G., Stiehl, A., 1990. Ursodeoxycholate reduces hepatotoxicity of bile salts in primary human hepatocytes. Hepatology 12, 486-491. 
Gores, G.J., Miyoshi, H., Botla, R., Aguilar, H.I., Bronk, S.F., 1998. Induction of the mitochondrial permeability transition as a mechanism of liver injury during cholestasis: a potential role for mitochondrial proteases. Biochim. Biophys. Acta 1366, 167-175.

Greim, H., Trulzsch, D., Czygan, P., Rudick, J., Hutterer, F., Schaffner, F., Popper, H., 1972. Mechanisms of cholestasis. 6. Bile acids in human livers with or without biliary obstruction. Gastroenterology 63, 846-850.

Heuman, D.M., Pandak, W.M., Hylemon, P.B., Vlahcevic, Z.R., 1991. Conjugates of ursodeoxycholic acid protect against cytotoxicity of more hydrophobic bile salts: in vitro studies in rat hepatocytes and human erythrocytes. Hepatology 14, 920-926.

Hillaire, S., Ballet, F., Franco, D., Setchell, K.D., Poupon, R., 1995. Effects of ursodeoxycholic acid and chenodeoxycholic acid on human hepatocytes in primary culture. Hepatology 22, 82-87.

Hofmann, A.F., 1984. Chemistry and enterohepatic circulation of bile acids. Hepatology 4, 4S-14S.

Kaplan, M., 1994. Primary biliary cirrhosis - a first step in prolonging survival. New Engl. J. Med. 330, 1368-1387.

Krahenbuhl, S., Stucki, J., Reichen, J., 1992. Reduced activity of the electron transport chain in liver mitochondria isolated from rats with secondary biliary cirrhosis. Hepatology $15,1160-1166$.

Krahenbuhl, S., Fischer, S., Talos, C., Reichen, J., 1994. Ursodeoxycholate protects oxidative mitochondrial metabolism from bile acid toxicity: dose-response study in isolated rat liver mitochondria. Hepatology 20, 1595-1601.

Lindor, K.D., Lacerda, M.A., Jorgensen, R.A., DeSotel, C.K., Batta, A.K., Salen, G., Dickson, E.R., Rossi, S.S., Hofmann, A.F., 1998. Relationship between biliary and serum bile acids and response to ursodeoxycholic acid in patients with primary biliary cirrhosis. Am. J. Gastroenterol. 93, 1498-1504.

Mitsuyoshi, H., Nakashima, T., Sumida, Y., Yoh, T., Nakajima, Y., Ishikawa, H., Inaba, K., Sakamoto, Y., Okanoue, T., Kashima, K., 1999. Ursodeoxycholic acid protects hepatocytes against oxidative injury via induction of antioxidants. Biochem. Biophys. Res. Commun. 263, $537-542$.

Moran, J.H., Schnellmann, R.G., 1996. A rapid beta-NADHlinked fluorescence assay for lactate dehydrogenase in cellular death. J. Pharmacol. Toxicol. Methods 36, 41-44.

Muller, M., Jansen, P.L.M., 1997. Molecular aspects of hepatobiliary transport. Am. J. Physiol. 272, G1285-G1303.

Neuberger, J., 2000. URSO - panacea or placebo? Hepatology 31, 1027-1028.

Pazzi, P., Puviani, A.C., Dalla Libera, M., Guerra, G., Ricci, D., Gullini, S., Ottolenghi, C., 1997. Bile salt-induced cytotoxicity and ursodeoxycholate cytoprotection: in-vitro study in perifused rat hepatocytes. Eur. J. Gastroenterol. Hepatol. 9, 703-709.
Plaa, G.L., de Lamirande, E., Lewittes, M., Yousef, I.M., 1982. Liver cell plasma membrane lipids in manganesebilirubin-induced intrahepatic cholestasis. Biochem. Pharmacol. 31, 3698-3701.

Poupon, R.E., Poupon, R., Balkau, B., 1994. Ursodiol for the long-term treatment of primary biliary cirrhosis. The UDCA-PBC study group. New Engl. J. Med. 330, 13421347.

Que, F.G., Phan, V.A., Phan, V.H., LaRusso, N.F., Gores, G.J., 1999. GUDC inhibits cytochrome c release from human cholangiocyte mitochondria. J. Surg. Res. 83, 100105.

Rodrigues, C.M.P., Fan, G., Ma, X., Kren, B.T., Steer, C.J., 1998. A novel role for ursodeoxycholic acid in inhibiting apoptosis by modulating mitochondrial membrane perturbation. J. Clin. Invest. 101, 2790-2799.

Rolo, A.P., Oliveira, P.J., Moreno, A.J.M., Palmeira, C.M., 2000. Bile acids affect liver mitochondrial bioenergetics: possible relevance for cholestasis therapy. Toxicol. Sci. 57, $177-185$.

Rust, C., Karnitz, L.M., Paya, C.M., Moscat, J., Simari, R.D., Gores, G.J., 2000. The bile acid taurochenodeoxycholate activates a phosphatidylinositol 3-kinase-dependent survival signaling cascade. J. Biol. Chem. 275, 20210-20216.

Sarbu, C., Kuhajada, K., Keveresan, S., 2001. Evaluation of the lipophilicity of the bile acids and their derivatives by thin-layer chromatography and principal component analysis. J. Chromatogr. A 917, 361-366.

Schaffner, F., Bacchin, P.G., Hutterer, F., Scharnbeck, H.H., Sarkozi, L.L., Denk, H., Popper, H., 1971. Mechanisms of cholestasis. 4. Structural and biochemical changes in the liver and serum in rats after bile duct ligation. Gastroenterology $60,888-897$.

Scholmerich, J., Becher, M.S., Schmidt, K., Schubert, R., Kremer, B., Feldhaus, S., Gerok, W., 1984. Influence of hydroxilation and conjugation of bile salts on their membrane-damaging properties: studies on isolated hepatocytes and lipid membrane vesicles. Hepatology 4, 661-666.

Seglen, P.O., 1976. Preparation of isolated rat liver cells. Methods Cell. Biol. 13, 29-83.

Sokol, R.J., Devereaux, M., Khandwala, R., O'Brien, K., 1993. Evidence for involvement of oxygen free radicals in bile acid toxicity to isolated rat hepatocytes. Hepatology 17, 869-881.

Spivey, J.R., Bronk, S.F., Gores, G.J., 1993. Glycochenodeoxycholate-induced lethal hepatocellular injury in rat hepatocytes. Role of ATP depletion and cytosolic free calcium. J. Clin. Invest. 92, 17-24.

van Hoogstraten, H.J., Hansen, B.E., van Buuren, H.R., tem Kate, F.J., van Berge-Henegouwen, G.P., Schalm, S.W., 1999. Prognostic factors and long-term effects of ursodeoxycholic acid on liver biochemical parameters in patients with primary biliary cirrhosis. Dutch Multi-Centre PBC Study Group. J. Hepatol. 31, 256-262. 